\title{
OBESITY
}

\section{Reduction in hepatic left lobe volume is a good indicator of metabolic improvement after bariatric surgery}

A recent study published in the International Journal of Obesity has demonstrated that a reduction in hepatic left lobe volume (HLLV) is the best predictor of improvement of various cardiometabolic risk factors in obese women undergoing gastric banding. previously developed an easy, safe
Ferruccio Santini and colleagues

and low-cost ultrasound technique to measure HLLV, and they found HLLV to be a sensitive indicator of ectopic fat deposition. BMI is commonly used to classify obesity, but it is not necessarily the best predictor of morbidities associated with abnormal fat accumulation.

Santini and colleagues investigated the relationship between several
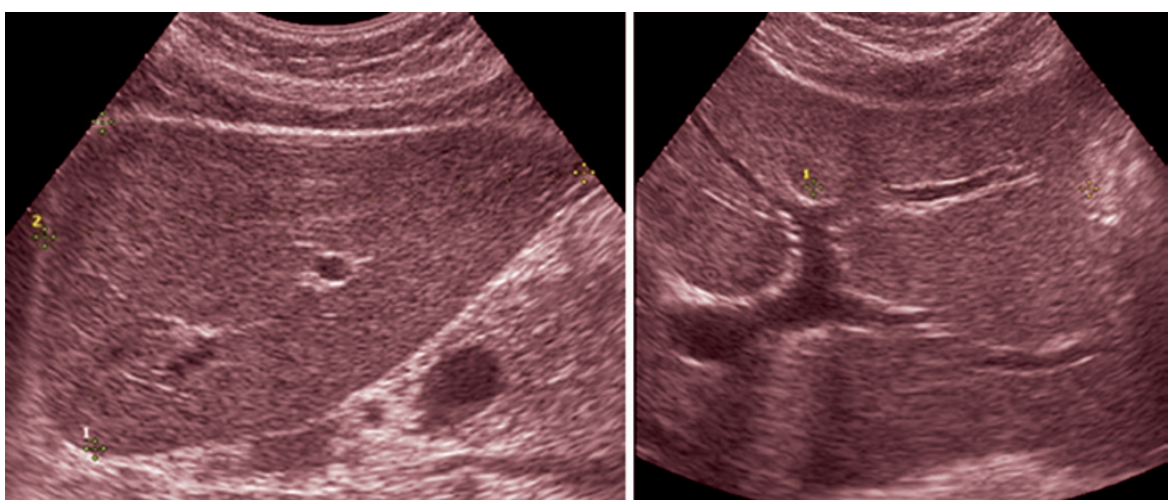

Longitudinal (left-hand panel) and axial (right-hand panel) ultrasound scans of the liver, used to calculate the hepatic left lobe volume. Courtesy of F. Santini. anthropometric measures (HLLV, body weight, BMI, subcutaneous and intraabdominal fat) and various indicators of the metabolic syndrome in 75 obese women before and after gastric banding.

Weight loss achieved by gastric banding was associated with a reduction in HLLV, and statistical analysis showed HLLV to cluster with parameters defining the metabolic syndrome. "The reduction of HLLV appeared as the best single predictor of improvement of various cardiometabolic risk factors," says Santini. He concludes, "A possible implication of our findings is the introduction of HLLV measurement into clinical practice as an easy and low-cost indicator of metabolic syndrome and cardiovascular risk."

\section{Isobel Franks}

Original article Giannetti, M. et al. Hepatic left lobe volume is a sensitive index of metabolic improvement in obese women after gastric banding. Int. J. Obes. doi:10.1038/ ijo.2011.243 